PROCEEDINGS OF THE

AMERICAN MATHEMATICAL SOCIETY

Volume 125, Number 9, September 1997, Pages 2527-2529

S 0002-9939(97)03918-X

\title{
THE RING OF GLOBAL SECTIONS OF MULTIPLES OF A LINE BUNDLE ON A TORIC VARIETY
}

\author{
E. JAVIER ELIZONDO
}

(Communicated by Ron Donagi)

\begin{abstract}
In this article we prove that for any complete toric variety, and for any Cartier divisor, the ring of global sections of multiples of the line bundle associated to the divisor is finitely generated.
\end{abstract}

\section{INTRODUCTION}

Let us start by stating the result and a few consequences. Let $X$ be an algebraic variety which is complete over a field $K$, and let $D$ be any effective Cartier divisor on $X$. We denote by $\mathcal{O}(n D)$ the line bundle associated to $n D$, with $n \geq 0$. It is a very interesting problem to know if the ring

$$
R:=\bigoplus_{n \geq 0} H^{0}(X, \mathcal{O}(n D))
$$

is a finitely generated $K$-algebra. It was O. Zariski who worked out the case of algebraic surfaces in order to solve the Riemann-Roch problem, and gave examples where $R$ is not finitely generated, see [Zar62]. In this article we prove that the ring $R$ is finitely generated when $X$ is a complete toric variety (perhaps singular).

The ring $R$ appears in many interesting problems, for example, if $X$ is a nonsingular projective variety, then the finite generation of $R$ implies that the series

$$
\sum_{n \geq 0} \operatorname{dim} H^{0}(X, \mathcal{O}(n D)) t^{n}
$$

is rational. It was asked by S. D. Cutkosky and V. Srinivas [CS93] if this series is rational when $D$ is a nef divisor. Our result gives trivially a positive answer in the case of a complete toric variety, and $D$ any effective Cartier divisor. Furthermore, the rationality of the series also allows us to compute, at least theoretically, the dimension of $H^{0}(X, \mathcal{O}(n D))$ in terms of $n$. This is the Riemann-Roch problem; see for example [Zar62] and [CS93]. Observe that these dimensions are not given by the Riemann-Roch theorem since the variety $X$ can be singular, and the line bundle $\mathcal{O}(n D)$ is not necessarily generated by its global sections. We would like to make a final remark. The ring $R$ is a subring of the ring $S=\bigoplus_{D>0} H^{0}(X, \mathcal{O}(D))$, which is finitely generated in the case of toric varieties. In fact, D. Cox proves in [Cox95]

Received by the editors March 14, 1996.

1991 Mathematics Subject Classification. Primary 14C20, 14M25.

Supported in part by grant CONACYT 3936-E, and DGAPA IN101296.

(C) 1997 American Mathematical Society 
that the ring $S$ is a polynomial ring graded by the the monoid of effective divisors classes in the Chow group $A_{k-1}(X)$ of $X$, where $k$ is the dimension of $X$.

\section{The RING OF GLOBAL SECTIONS}

In this section we prove that the ring $R$, which was defined by equation (1), is a finitely generated $K$-algebra. Throughout this section $X$ means a complete toric variety over a field $K$, and $D$ a Cartier divisor in $X$. The construction of the cone $C_{R}$, in the proof of the Theorem, is a well known construction; see for example [Bat93]. We start by recalling an important lemma that will be used in the proof of the theorem.

Lemma 2.1 (Gordan). If $\sigma$ is a strongly convex rational polyhedral cone, and $\sigma^{\vee}$ its dual, then $S_{\sigma}=\sigma^{\vee} \cap M$ is a finitely generated semigroup.

Now, we are ready for the main result.

Theorem 2.2. Let $X$ be a complete toric variety, perhaps singular, and let $D$ be a Cartier divisor in $X$. Then the ring

$$
R:=\bigoplus_{n \geq 0} H^{0}(X, \mathcal{O}(n D))
$$

is finitely generated as a $K$-algebra.

Proof. We first observe that we can consider our divisor $D$ to be $T$-invariant. For the following sequence is exact (see [Dan78, page 116])

$$
0 \longrightarrow M \longrightarrow \operatorname{Div}_{\mathrm{T}} X \longrightarrow \operatorname{Pic}(X) \longrightarrow 0
$$

where $\operatorname{Div}_{\mathrm{T}} X$ is the group of $T$-invariant Cartier divisors and $M:=\operatorname{Hom}_{\mathbb{Z}}(N, \mathbb{Z})$ is the dual lattice of $N \cong \mathbb{Z}^{k}$. Then we can write $D$ as $D=\sum_{i=1}^{s} a_{i} D_{i}$ with $\left\{D_{i}\right\}$ the set of invariant divisors. We consider the convex rational polyhedron $P_{n D}$ in $M_{\mathbb{Q}}:=M \bigotimes_{\mathbb{Z}} \mathbb{Q}$ defined as

$$
P_{n D}=\left\{u \in M_{\mathbb{Q}} \mid\left\langle u, v_{i}\right\rangle \geq-n a_{i} \text { for all } i\right\}
$$

where $v_{i}$ is the the first element in the lattice appearing in the divisor $D_{i}$. We know that generators for the space $H^{0}(X, \mathcal{O}(n D))$ are given by the elements of $P_{n D} \cap M$, which is a finite set because $X$ is a complete variety; this also implies that $P_{D}$ is a rational convex polytope. Let us embed the $k$-dimensional $\mathbb{Q}$-vector space $M_{\mathbb{Q}}$ into the $(k+1)$-dimensional $\mathbb{Q}$-vector space $M_{\mathbb{Q}}^{k+1}:=\mathbb{Z}^{k+1} \mathbb{Q}_{\mathbb{Z}} \mathbb{Q}$ as the hyperplane with equation $x_{k+1}=1$, where $\left(x_{1}, \ldots, x_{k+1}\right)$ are coordinates for $M_{\mathbb{Q}}^{k+1}$. Denote by $C_{R}$ the $(k+1)$-dimensional cone in $M_{\mathbb{Q}}^{k+1}$ generated by the rays starting at the origin and passing through the vertices of $P_{D}$ (see Figure 1).

It follows from the definition that $P_{n D}=n P_{D}$, and this implies that the intersection of the hyperplane $x_{k+1}=n$ with the cone $C_{R}$ is just the polyhedron $P_{n D}$. In other words, the cone $C_{R}$ is the cone associated to the ring $R$, in the sense that any element of the ring $R$ is a finite linear combination of integral points of $C_{R} \cap \mathbb{Z}^{k+1}$. The theorem follows since the semigroup $C_{R} \cap \mathbb{Z}^{k+1}$ is finitely generated by Lemma 2.1 (Gordan). 


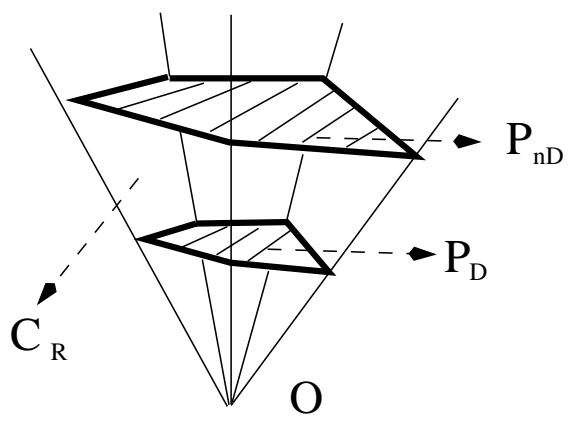

Figure 1. The cone $C_{R}$

\section{ACKNOWLEDGEMENT}

I thank very much V. Srinivas for suggesting this problem to me and for many wonderful and fruitful conversations. I also would like to thank A. King for bringing to my attention the article of D. Cox, and to the first referee for pointing out the article of V. Batyrev.

\section{REFERENCES}

[Bat93] Victor V. Batyrev. Variations of the mixed hodge structure of affine hypersurfaces in algebraic tori. Duke Math. J., 69(2):349-409, Feb 1993. MR 94m:14067

[Cox95] David A. Cox. The homogeneous coordinate ring of a toric variety. J. Algebraic Geom., 4(3):17-50, 1995. MR 95i:14046

[CS93] S. D. Cutkosky and V. Srinivas. On a problem of Zariski on dimensions of linerar systems. Ann. of Math., 137:531-559, 1993. MR 94g:14001

[Dan78] V. I. Danilov. The geometry of toric varieties. Russian Math. Surveys, 33(2):97-154, 1978. MR 80g:14001

[Zar62] O. Zariski. The theorem of Riemann-Roch for high multiples of an effective divisor on an algebraic surface. Ann. of Math., 76:560-616, 1962. MR 25:5065

Instituto de Matemáticas, UNAM, Ciudad Universitaria, México D.F. 04510

E-mail address: javier@math.unam.mx 